\title{
Las políticas territoriales de desarrollo rural de la Unión Europea: un balance de veinte años en Castilla y León
}

\author{
PABLO GORDO GÓMEZ \\ Departamento de Economía Aplicada, UNIVERSIDAD DE VALLADOLID, ESPAÑA. E- \\ mail: pablog@emp.uva.es
}

\begin{abstract}
RESUMEN
La política específicamente agraria se ha mostrado insuficiente para dinamizar los espacios rurales de Castilla y León, cuyas características físicas y estructurales introducen limitaciones para la producción en el marco competitivo de la Unión Europea. Ante esta realidad, las políticas territoriales de desarrollo rural se presentan como la alternativa para aumentar la competitividad, apoyando la reconversión, diversificando las actividades económicas mediante el recurso al potencial endógeno y contribuyendo a fijar la población y mejorar la calidad de vida de los diferentes espacios rurales. El balance de la aplicación de las distintas actuaciones bajo el enfoque LEADER, durante las dos últimas décadas, ha sido: la promoción y desarrollo del turismo, con diversas opciones; $\mathrm{y}$, el impulso de pequeñas y medianas empresas (PYMEs), que han contribuido a la transformación y comercialización de productos agrarios y a generar empleo. Sin embargo, este impacto se ha verificado en pocos núcleos de mayor tamaño, cuyo efecto difusor ha sido limitado para reducir los desequilibrios del espacio rural regional y no ha logrado participar activamente en redes europeas de cooperación transnacional.
\end{abstract}

Palabras clave: Políticas territoriales, Desarrollo rural, Unión Europea, LEADER, Castilla y León.

\section{The Territorial Rural Development Policies of the European Union: a Twenty-Year Balance in Castilla y León}

\begin{abstract}
Specific farm policies have not revitalized Castile and Leon rural environments, whose physical and structural characteristics limit their productive possibilities in the EU competitive market. Faced with this situation territorial polices for rural developments have been considered adequate for enhancing competitiveness by helping restructuring, diversifying economic activities emphasising endogenous potentialities, maintaining population in rural areas, and increasing quality of life in them. An assessment of the results obtained within the LEADER framework in the last two decades indicates that rural tourism has benefited, and that there has been a boost for the farm activities, especially trade and marketing activities, which small and medium-sized enterprises (SMEs) have started and that have led to employment gains. However, the benefits have been located in few places, only those that boast high sizes, and have not achieved a visible reduction of rural inequalities nor have translated in an active participation in European networks of multinational cooperation.
\end{abstract}

Keywords: Land Policies, Rural Development, European Union, LEADER, Castilla y León

Clasificación JEL: R58, O13, O18

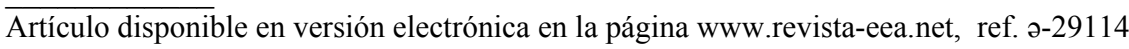




\section{INTRODUCCIÓN}

La existencia de amplios espacios rurales en la Unión Europea que registran pérdidas continuas de población y de actividad económica, con importantes repercusiones en términos de desigualdad espacial en la distribución de la renta y en la conservación del patrimonio y del medio ambiente, ha dado lugar a la aplicación de una serie de políticas territoriales orientadas a facilitar la transformación y reconversión del mundo rural propiciando en él una tipología de actividades económicas más demandadas por la población, de forma que éstas puedan contribuir a la generación de renta y empleo, además de sustituir paulatinamente a las producciones agrarias tradicionales, muchas de las cuales actualmente se mantienen gracias a las subvenciones de la Política Agrícola Común (PAC) y, en el futuro, no ofrecen perspectivas de rentabilidad ante el irreversible proceso de globalización registrado en los mercados mundiales. En este contexto, Castilla y León es una de las regiones de la Unión Europea más afectadas, puesto que cuenta con una elevada proporción de espacio rural en su territorio, cuyo declive económico y poblacional resulta especialmente significativo.

El presente estudio ${ }^{1}$ se enmarca en el contexto socioeconómico que caracteriza el espacio rural de Castilla y León, cuyos municipios vienen registrando importantes diferencias en cuanto al grado de desarrollo económico alcanzado y a las perspectivas que tienen ante el futuro. El objetivo principal del análisis realizado consiste en valorar la repercusión que ha podido tener la política territorial que, bajo directrices europeas, ha venido aplicándose en el espacio regional desde la década de los noventa hasta la actualidad. Todo ello con la finalidad de que el conocimiento de esta realidad rural, así como los resultados que las acciones de desarrollo emprendidas están teniendo sobre el territorio, puedan servir de referencia para orientar adecuadamente el uso de este tipo de políticas durante los próximos años.

\section{CONTEXTO SOCIOECONÓMICO DEL ESPACIO RURAL REGIONAL}

La Comunidad Autónoma de Castilla y León es una de las regiones más extensas de la Unión Europea, con una superficie de $94.226 \mathrm{~km}^{2}$ que representa el $18,6 \%$ del territorio nacional. Se trata de una región interior que, en su

\footnotetext{
${ }^{1}$ Algunas consideraciones sobre este tema han sido analizadas por el autor en otros trabajos. Los lectores interesados pueden encontrar información más ampliada en: Instituto de Estudios Europeos (2007): "Perspectivas económicas de los espacios rurales de Castilla y León: Experiencia de las políticas territoriales de desarrollo rural aplicadas y propuestas para el período 2007-2013". Capítulo del libro titulado: Tratado de Roma en su 50 aniversario (19572007). Balance socioeconómico de la Unión Europea. Valladolid. Universidad de Valladolid (pp. 687-708). ISBN 978-84-9836-224-4.
} 
conjunto, constituye una cuenca sedimentaria rodeada por bordes montañosos alcanzando una altitud media de unos 800 metros sobre el nivel del mar, de forma que sus características geomorfológicas limitan en cierta medida su desarrollo, máxime al estar localizada en uno de los extremos, el S.W., de la Unión Europea, erigiéndose como región de un país periférico con un clima de tipo mediterráneo interior del que se derivan elevados contrastes de temperatura y una pluviometría escasa que, a su vez, presenta importantes desigualdades espaciales y estacionales. Estas condiciones climáticas condicionan de forma importante unas producciones agrarias que coinciden con las grandes producciones europeas de cereales, carne de bovino, leche y azúcar, las cuales se están viendo seriamente afectadas por los sucesivos procesos de reforma de la PAC que, sin duda, van a continuar a lo largo de los próximos años.

La población de Castilla y León, 2.510 .849 habitantes según el Padrón Municipal correspondiente a 2010, representa solamente el 5,4\% de la española, siendo su densidad 27,1 hab $/ \mathrm{km}^{2}$, frente a los $92,9 \mathrm{hab} / \mathrm{km}^{2}$ de España. Esta Comunidad Autónoma tiene un total de 2.248 términos municipales, de los cuales 2.204 presentan un marcado carácter rural; suponen el $96 \%$ de la superficie regional y en ellos vive más del $41 \%$ de la población, cuya densidad solamente alcanza los $11,5 \mathrm{hab} / \mathrm{km}^{2}$. Esta dispersión de los pobladores y el alto grado de envejecimiento que caracteriza al medio rural de Castilla y León ponen claramente de manifiesto las dificultades de aplicación de cualquier estrategia de desarrollo en un territorio que, paulatinamente, va presentando unos síntomas de desertización poblacional que resultan especialmente significativos en estos espacios rurales. Si bien es cierto que en los últimos años la incorporación de población inmigrante a muchos municipios rurales de Castilla y León podría llegar a contribuir a su regeneración demográfica, tampoco debemos olvidar que se trata de una población poco numerosa e inestable que, en la mayoría de los casos, accede al mercado laboral nacional a través de las actividades del sector agrario $^{2}$ pero que, en cuanto tiene la mínima posibilidad, se desplaza hacia ocupaciones relacionadas principalmente con el sector terciario.

Por otro lado, aunque el sector primario ha perdido peso de forma continuada a lo largo de la última década, la actividad agraria en Castilla y León representa todavía una participación destacada dentro de la estructura sectorial y regional; así, la proporción de valor añadido sobre el conjunto de la economía regional es superior al 6\% del total, dando empleo a más de 72.000 personas que suponen el $6 \%$ de la población activa regional. Las principales producciones son: cereales, carne, leche y azúcar, sometidas todas ellas a importantes cambios debido a la considerable influencia del continuo proceso de globalización que se está registrando en los mercados mundiales. Junto al

\footnotetext{
${ }^{2}$ En diciembre de 2009 existían en Castilla y León 58.898 afiliados extranjeros a la Seguridad Social, de los que 7.463 (el 12,7\%) lo estaban en el Régimen Especial Agrario.
} 
protagonismo de estas materias primas, coincidente con las grandes producciones agrarias europeas de carácter excedentario, debe tenerse en cuenta, además, que el tamaño medio de las explotaciones de la región es aún muy reducido aunque en los últimos años se ha logrado superar la media de 50 hectáreas por explotación.

En definitiva, este espacio rural regional continuará sometido en el futuro a un fuerte proceso de reconversión $\mathrm{y}$, a la vez, soportará el paulatino decrecimiento y envejecimiento poblacional en los núcleos de menor tamaño y una tendencia migratoria de los individuos hacia municipios más grandes, con mejores alternativas de empleo, calidad de los servicios y nivel de desarrollo. Estos flujos de población han determinado que el medio rural esté habitado, fundamentalmente, por familias de edad avanzada cuya fuente de ingresos se ha visto reducida debido a las jubilaciones y prejubilaciones, circunstancia que se convierte en un freno adicional al desarrollo, tanto por la disminución del potencial de trabajo, como por los cuantiosos recursos destinados a facilitar servicios sociales a la población de la región con la media de edad más alta de la Unión Europea.

Según se ha indicado anteriormente, el espacio rural regional es muy extenso y afecta, además, a un elevado número de municipios; por esta razón, resulta de gran interés tener muy presente la diferente caracterización de éstos, dado el importante condicionante que estas circunstancias pueden suponer para la aplicación de las políticas territoriales de desarrollo rural. Partiendo de las consideraciones realizadas en algunos de nuestros trabajos de investigación (Fariñas Gómez; Gordo Gómez; Ríos Rodicio y Rodríguez Prado, 2004), cuya finalidad era determinar el perfil socioeconómico de esos núcleos de población a partir de las diferencias entre los distintos espacios rurales de la región, los análisis efectuados nos permitieron clasificar los municipios de Castilla y León menores de 15.000 habitantes en siete categorías de municipios rurales que fueron denominados del siguiente modo:

- Municipios agrarios en declive.

- Municipios agrarios que proporcionan mano de obra.

- Municipios agrarios en declive que mantienen equipamientos públicos.

- Municipios de elevado tamaño, valor patrimonial y dotación turística.

- Municipios con elevado tamaño y dinamismo económico propio.

- Municipios periféricos con potencial turístico.

- Municipios periurbanos residenciales.

Ante esta diversidad económica de nuestro mundo rural regional, las políticas de desarrollo aplicadas tradicionalmente se han dirigido, de forma 
prioritaria, a mantener las rentas de los agricultores mediante ayudas directas y a mejorar las condiciones productivas del sector agrario, en lugar de incidir en la reconversión decidida de éste, orientando nuevas actividades económicas que partan de un uso del espacio agrario distinto de los aprovechamientos tradicionales. Así, en los últimos años, se ha podido constatar que la política específicamente agraria, encaminada a aumentar la competitividad del sector, no es una solución suficiente para intentar solucionar los problemas de estas áreas, de forma que se está produciendo una revisión de las actuaciones, incidiendo en la necesidad de diversificar las actividades económicas que se localizan en ellas.

No cabe duda que, ante la diferente tipología de municipios existente en el espacio rural regional, se pone claramente de manifiesto la necesidad de que las políticas de desarrollo propuestas sean diseñadas en función del espacio específico sobre el que van a actuar. En consecuencia, es preciso poner en cuestión las actuaciones territoriales basadas en criterios de igualitarismo, ya que, si bien ello puede resultar políticamente sencillo, sus logros van a ser muy limitados, porque para un elevado número de municipios sería muy difícil obtener rendimientos importantes de las políticas de desarrollo rural propuestas desde la Unión Europea.

La situación socioeconómica del mundo rural de Castilla y León sigue siendo, actualmente, bastante complicada pero, a su vez, y en contrapartida, el campo de aplicación de programas de desarrollo rural integrado es muy amplio, tanto por el escenario en el que se inscriben como por sus potencialidades de desarrollo. Dadas las condiciones de las que parte su territorio, puede apreciarse lo decisivo que resulta tratar de favorecer los factores de desarrollo en esta región, cuyo problema fundamental es el peligro de desertización económica y poblacional con efectos irreversibles sobre el territorio, tanto en términos sociales como medioambientales. De modo que el objetivo de mantener la población en el territorio y dinamizar los potenciales de actividad debe ser considerado como uno de los el ejes básicos de las políticas territoriales de desarrollo rural aplicadas en esta Comunidad Autónoma a lo largo de los últimos años.

\section{LAS POLÍTICAS TERRITORIALES DE DESARROLLO RURAL DE LA UNIÓN EUROPEA: LA EXPERIENCIA DE CASTILLA Y LEÓN}

Uno de los grandes objetivos de la política de desarrollo rural consiste en aumentar la competitividad del sector agrario mediante el apoyo a su reconversión. No obstante, debemos considerar también dentro de esta política un tipo concreto de actuaciones orientadas a mejorar la calidad de vida de las áreas rurales a través de la diversificación de actividades económicas. En este 
sentido, la Iniciativa Comunitaria LEADER I constituyó hace dos décadas una interesante propuesta de política de desarrollo rural aplicada desde la Comisión Europea con sello propio e independiente. Con un presupuesto inicialmente muy modesto, se trataba de apoyar la transformación del mundo rural a través de la orientación del espacio agrario para usos distintos a los tradicionales, propiciando con ello la existencia en esas áreas de los denominados Grupos de Acción Local (GAL), asociaciones sin ánimo de lucro integradas por los propios agentes económicos y sociales procedentes del mismo territorio para, a través de ellos, poner en marcha programas de desarrollo de carácter multisectorial e integrado, acordes con las particularidades de cada zona, apoyando también diversas actividades distintas a las típicamente agrarias, tales como la valorización de la transformación alimentaria, el turismo rural, las pequeñas empresas, la artesanía y diversos servicios, así como otras potencialidades del territorio ligadas a sus valores patrimoniales, culturales y medioambientales. Todo ello sin olvidar la importante labor de difusión realizada por los $G A L$ sobre este tipo de programas en numerosos municipios, así como el apoyo a una formación profesional específica orientada a las necesidades de desarrollo de las zonas donde se actuaba. Los resultados de esta Iniciativa resultan difíciles de valorar cuantitativamente, pero no cabe duda que constituyó en su día un cambio de rumbo muy significativo en la política de desarrollo rural aplicada hasta entonces (Tabla 1).

Tabla 1

Políticas territoriales de desarrollo rural aplicadas en Castilla y León (1991-2013)

\begin{tabular}{|c|c|c|c|c|c|c|c|}
\hline Periodo & Programa & $\begin{array}{c}\text { Superficie } \\
\mathbf{( k m}^{2} \mathbf{)}\end{array}$ & $\begin{array}{c}\text { Población } \\
\text { (Habitantes) }\end{array}$ & $\begin{array}{c}\text { Número de } \\
\text { municipios }\end{array}$ & $\begin{array}{c}\text { Número } \\
\text { de GAL }\end{array}$ & $\begin{array}{c}\text { Gasto } \\
\text { Público } \\
\text { (millones } \\
\text { de euros) }\end{array}$ & $\begin{array}{c}\text { Empleo } \\
\text { generado }\end{array}$ \\
\hline $1991-1993$ & LEADER I & 9.848 & 110.737 & 218 & 6 & 56 & s.d. \\
\hline \multirow{2}{*}{$1995-2000$} & LEADER II & 30.608 & 324.614 & 682 & 16 & 108 & 1.475 \\
\cline { 2 - 8 } & PRODER & 38.161 & 495.303 & 960 & 21 & 100 & 2.077 \\
\hline \multirow{2}{*}{$2000-2007$} & LEADER+ & 34.789 & 290.731 & 749 & 17 & 100 & 1.362 \\
\cline { 2 - 8 } & PRODERCAL & 54.893 & 759.150 & 1.432 & 27 & 153 & 2.095 \\
\hline $2007-2013$ & LEADERCAL & 91.951 & 1.043 .902 & 2.204 & 44 & 215 & $585^{\star}$ \\
\hline
\end{tabular}

* Datos estimados a 30 de junio de 2010.

Fuente: Elaboración propia a partir de los diferentes Informes de Evaluación realizados a lo largo de estos años.

Es indudable que uno de los aspectos más positivos de la Iniciativa LEADER $I$ fue el de sentar el precedente de un nuevo enfoque para el desarrollo rural, facilitando de este modo las condiciones para la puesta en marcha, a partir de 1995, de la Iniciativa Comunitaria LEADER II, el Programa Operativo de Desarrollo y Diversificación Económica de Zonas Rurales (PRODER), así 
como la Iniciativa LEADER+ y el Programa PRODERCAL en el año $2000 \mathrm{y}$, más recientemente, el Programa LEADERCAL, denominación que recibe en Castilla y León la aplicación del Enfoque LEADER correspondiente al Eje 4 del Programa de Desarrollo Rural de Castilla y León 2007-2013. Todas estas acciones mantienen unos objetivos fundamentados en la revitalización de amplias zonas rurales del territorio de la Comunidad Autónoma, aprovechando los procesos económicos que se apoyan en recursos endógenos y en una estrategia innovadora que permita encontrar soluciones a los problemas de los espacios rurales de la región. Bajo este método, se han llegado a poner en marcha un total de 44 Grupos de Acción Local con una disposición de fondos públicos cuatro veces superior a la asignada inicialmente, afectando, de esta forma, a más de 2.000 municipios de la región y, con ello, a la mayor parte del territorio rural regional (Tabla 1).

\subsection{Aplicación de la Iniciativa Comunitaria LEADER II y el Programa PRODER. Período 1995-2000}

En el caso de la Iniciativa Comunitaria LEADER II y el Programa PRODER los objetivos que con mayor frecuencia fueron planteados estaban siempre relacionados con el mantenimiento de la población en el territorio, el incremento de la base productiva local, la mejora de la calidad de vida y la consolidación de grupos de desarrollo capaces de movilizar a los agentes sociales en el territorio, estableciendo una sede permanente en cada una de las 37 comarcas (16 LEADER y 21 PRODER) acogidas a estos Programas con la finalidad de facilitar la orientación y tramitación de los proyectos de inversión seleccionados. Los recursos más valorados por los Grupos de Acción Local sobre los que apoyaban su estrategia de desarrollo, fueron, en la mayoría de los casos, el turismo y el patrimonio cultural y arquitectónico; sin embargo, los problemas de despoblación y envejecimiento demográfico permanecieron latentes en todo el territorio y el inicio de este proceso de revitalización económica, en algunos municipios, no logró introducir cambios importantes en su dinámica poblacional.

Las principales acciones innovadoras de la Iniciativa LEADER $I I^{3}$ y del Programa PRODER, durante el periodo 1995-2000, fueron la oferta de nuevos productos y servicios y la búsqueda de nuevos mercados y clientes. El mayor freno a la innovación se observaba siempre en la ausencia de promotores derivada de las ya referidas condiciones socioeconómicas de la mayoría de los

\footnotetext{
${ }^{3}$ A este respecto puede consultarse el documento titulado Evaluación "Ex-post" de la ejecución del Programa Regional de Castilla y León en la Iniciativa Comunitaria LEADER II (19951999), dirigido por el autor de este trabajo y llevado a cabo con la colaboración de los siguientes profesores del Departamento de Economía Aplicada de la Universidad de Valladolid: Belén Miranda Escolar, José Antonio Salvador Insúa y José Luis Rojo García.
} 
territorios en los que actuaban los GAL. Los resultados globales mostraron, no obstante, un cierto nivel de innovación para el conjunto de las acciones desarrolladas, en relación con el tipo de actividad económica y social existente en su propio territorio antes de la aplicación de estos Programas.

Los efectos generales sobre el carácter multisectorial e integrado de las acciones llevadas a cabo supusieron un paso importante ante las peculiaridades socioeconómicas existentes en cada territorio. Los agentes que protagonizaron, generalmente de forma coordinada, cierto tipo de acciones fueron las pequeñas empresas y los profesionales del turismo, siendo los principales responsables de impulsar este tipo de iniciativas los equipos técnicos de los $G A L$ y los propios promotores de proyectos. Los obstáculos que frecuentemente impedían o dificultaban el desarrollo de acciones de carácter multisectorial procedían del fuerte individualismo de sus protagonistas, siendo ésta una característica bastante extendida en el medio rural de Castilla y León.

Los efectos más positivos de la participación de los distintos colectivos sociales representados en los $G A L$ fueron el estímulo para nuevos proyectos y el efecto demostrativo transmitido a otros programas de desarrollo en áreas próximas. En cuanto a la participación de los socios en los órganos de decisión de los GAL, ya se empezó a percibir claramente la posición dominante de los ayuntamientos sobre los demás socios, puesto que su representación, en términos generales, superaba a la que tenían en los órganos consultivos. Asimismo, la utilización de redes asociativas de carácter regional y nacional por los $G A L$ de la región tuvo, también, un efecto positivo, mejorando a lo largo del tiempo y observándose, a su vez, una progresiva participación de los $G A L$ en redes temáticas, donde casi todos ellos llegaron a mostrarse bastante activos. Sin embargo, no se consolidó suficientemente la participación en redes europeas y en proyectos de cooperación transnacional por parte de la mayoría de los $G A L$, ni se lograron resultados cuantitativos apreciables.

En términos económicos, los resultados de mayor impacto en la aplicación de la Iniciativa LEADER II y del Programa PRODER en la región fueron la promoción y desarrollo del turismo rural y las ayudas a la creación de pequeñas y medianas empresas (PYMEs). El elevado presupuesto dedicado a estas dos medidas contribuyó a mejorar la oferta turística de la región y a dinamizar un buen número de PYMEs no agrarias en el medio rural, con los consiguientes efectos positivos sobre el empleo, tanto directo como indirecto. Por el contrario, la valorización y comercialización de la producción agraria no respondió totalmente a las grandes expectativas generadas, por el hecho de constituir una de las claves del futuro desarrollo de la región; no obstante, la repercusión de estos Programas ha tenido resultados muy positivos para bastantes Grupos, tanto en la creación de empleo como en la consolidación de este tipo de actividades en sus respectivos territorios. En cuanto a las actividades relativas a 
la conservación y mejora del entorno medioambiental en las áreas rurales, por lo general, respondieron a las iniciativas de embellecimiento de los municipios por parte de los ayuntamientos, no llegando a tener efectos significativos sobre el empleo; si bien es cierto que una parte de estas actuaciones consiguieron llegar a repercutir positivamente en algunos proyectos de inversión relacionados con el turismo.

En definitiva, los efectos generales que la Iniciativa LEADER II y el Programa PRODER, correspondientes al período 1995-2000, tuvieron sobre el territorio han sido bastante positivos, llegando a generar más de 3.500 puestos de trabajo, logrando una mayor cohesión interna de la región ante el problema de sus áreas rurales y una mejora de la imagen local, así como la introducción de innovaciones y una utilización más eficiente de los recursos locales. En cambio, no consiguió calar lo suficiente, en el conjunto de los $G A L$, la apertura hacia la globalidad y el reforzamiento de los intercambios de experiencias con otros Estados Miembros, debido, básicamente, a la falta de precedentes de este tipo de actuaciones en el medio rural de Castilla y León.

\subsection{Resultados de la Iniciativa LEADER+ y del Programa PRODERCAL. Período 2000-2006}

En los Programas $L E A D E R+{ }^{4}$ y PRODERCAL ${ }^{5}$, que posteriormente se iniciaron en esta Comunidad Autónoma para la práctica totalidad de su espacio rural mediante la actuación de 44 Grupos de Acción Local, el objetivo básico fue mantener la población en el territorio, así como dinamizar su potencial de actividad; se pretendía impulsar así las iniciativas económicas vinculadas a las zonas rurales, a través de proyectos de inversión basados en la diversificación de actividades y mejora del entorno para facilitar la creación de empleo.

Las características de ambos Programas en Castilla y León correspondían básicamente al planteamiento general previsto en el Plan de Desarrollo Regional de esta Comunidad Autónoma para el período 2000-2006 y contemplaban los siguientes objetivos para las zonas rurales:

\footnotetext{
${ }^{4}$ La aprobación por la Comisión Europea del Programa LEADER+ (2000-2006) para Castilla y León, mediante Decisión de 20 de agosto de 2001, trataba de dar continuidad a la aplicación de las políticas de desarrollo rural iniciadas en esta Comunidad Autónoma, desde hacía más de una década, con la experiencia previa de la Iniciativa LEADER I (1991-1993) y, posteriormente, con la Iniciativa LEADER II (1994-1999) y el Programa PRODER (1995-1999).

${ }^{5}$ El Programa PRODERCAL (2000-2006) se puso en marcha en Castilla y León a través de la firma de diversos convenios, uno por cada Grupo de Acción Local. Éstos fueron rubricados por el Ministerio de Agricultura, Pesca y Alimentación, la Consejería de Agricultura y Ganadería de la Junta de Castilla y León y las distintas entidades a las que se les aprobó la condición de GAL. La firma de todos ellos se llevó a cabo el día 28 de octubre del 2002.
} 
- Impulsar el desarrollo endógeno sostenido en sus zonas de aplicación, a través de la diversificación, reestructuración y reconversión en el ámbito agrario en el medio rural.

- Mantener la población en el medio rural, frenando la regresión demográfica.

- Elevar las rentas y el bienestar de sus habitantes a niveles más próximos o equiparables a otras zonas más desarrolladas.

- Usar de forma sostenible los recursos naturales, procurando el aprovechamiento económico de las posibilidades que éstos ofrecen.

- Fomentar las inversiones que permitan la creación o el mantenimiento de puestos de trabajo y la potenciación y apoyo a las PYMEs.

A partir de estos objetivos, las principales líneas de actuación del Programa LEADER+ de Castilla y León siguieron una orientación acorde con los principios de la estrategia empleada para iniciar un tipo de desarrollo sostenible e integrado en las condiciones socioeconómicas del territorio, a través de un enfoque ascendente y participativo de los agentes que intervienen en el proceso y de su cooperación con otros territorios, buscando siempre la originalidad de sus intervenciones, así como la complementariedad y sinergias procedentes de otras acciones contempladas en el Plan de Desarrollo Regional y en el Programa Operativo Integrado de Castilla y León (POI) correspondiente al período (2000-2006). En el caso del Programa PRODERCAL, se integraron una serie de actuaciones, con una orientación similar a la Iniciativa $L E A D E R^{+}$, para propiciar el desarrollo endógeno de las zonas rurales, tal como se contemplaba, también, en el Programa Operativo de la Comunidad Autónoma, concretamente, dentro de las medidas ${ }^{6}$ 7.5. y 7.9. La primera se centraba en promover el desarrollo endógeno de las zonas rurales a través de las actividades agrarias, su diversificación y su reconversión, y estaba cofinanciada por la Sección de Orientación del Fondo Europeo de Orientación y Garantía Agraria (FEOGA-Orientación). La segunda, estaba orientada al desarrollo endógeno de las zonas rurales ligado a actividades no agrarias y la cofinanciación provenía del Fondo Europeo de Desarrollo Regional (FEDER). Con estos planteamientos, los elementos estratégicos que constituyeron las claves de aplicación de los Programas LEADER+ y PRODERCAL mantuvieron una clara coherencia con las condiciones socioeconómicas de la región y supusieron, además, una referencia importante para introducir los cambios y adaptaciones

\footnotetext{
${ }^{6}$ El Programa Operativo de Castilla y León 2000-2006 era plurifondo, recibía financiación de tres Fondos Estructurales (FEDER, FEOGA-Orientación y FSE), estaba integrado por 11 Ejes y lo conformaba un total de 50 medidas. Las medidas 7.5 y 7.9 se identificaban con la siguiente denominación: 7.5. Desarrollo endógeno de zonas rurales, relativo a las actividades agrarias y diversificación agraria y su reconversión, y 7.9. Desarrollo endógeno de las zonas rurales ligado a actividades no agrarias.
} 
necesarias con el fin de facilitar su desarrollo económico, en los que la iniciativa privada ostenta, indudablemente, el papel de protagonista principal.

Las actuaciones que emprendieron los Grupos de Acción Local en Castilla y León, hasta la finalización de los Programas $L E A D E R+$ y PRODERCAL, se agrupaban en una serie de ejes de intervención y medidas de desarrollo más específicas, referidas a las potencialidades del entramado productivo y social del conjunto del espacio rural. Estos Programas lograron generar una inversión total de 562 millones de euros, a lo largo de todo el período, en los diversos tipos de acciones financiadas, de los cuales el 59\% procedía de la iniciativa privada (Tabla 2).

Tabla 2

Recursos comprometidos en los programas LEADER+ y PRODERCAL al final del periodo de programación (2007)

\begin{tabular}{|c|c|c|c|c|}
\hline \multirow{2}{*}{ Medida de desarrollo } & \multicolumn{2}{|c|}{ LEADER+ } & \multicolumn{2}{|c|}{ PRODERCAL } \\
\hline & Euros & $\%$ S/Total & Euros & $\%$ S/Total \\
\hline Gastos de gestión y funcionamiento & 14.991 .768 & 7,35 & 22.235 .671 & 6,20 \\
\hline Servicios a la población & 18.127 .006 & 8,89 & 36.180 .218 & 10,09 \\
\hline Patrimonio Natural & 12.546 .744 & 6,15 & 21.389 .323 & 5,97 \\
\hline Valorización productos locales & 19.474 .374 & 9,55 & 40.908 .350 & 11,41 \\
\hline PYMES y servicios & 63.794 .597 & 31,27 & 89.046 .330 & 24,84 \\
\hline Patrimonio cultural y arquitectónico & 20.790 .034 & 10,19 & 35.176 .918 & 9,81 \\
\hline Turismo rural & 37.941 .421 & 18,60 & 73.953 .728 & 20,63 \\
\hline Formación y Empleo & 2.086 .861 & 1,02 & 2.174 .557 & 0,61 \\
\hline Cooperación & 10.243 .692 & 5,02 & 6.578 .500 & 1,84 \\
\hline Otras inversiones & 3.995 .404 & 1,96 & 30.840 .440 & 8,60 \\
\hline TOTAL & 203.991.901 & 100,00 & 358.484 .035 & 100,00 \\
\hline
\end{tabular}

Fuente: Dirección General de Infraestructuras y Diversificación Rural. Consejería de Agricultura y Ganadería. Junta de Castilla y León.

En los Informes de Evaluación Ex-post de la Iniciativa LEADER+ y de Seguimiento del Programa PRODERCAL realizados en Castilla y León, durante el período comprendido entre los años 2003 y $2008^{7}$, se trataron de valorar los

\footnotetext{
${ }^{7}$ Las consideraciones que se presentan en este apartado tienen como base los resultados del Informe de Evaluación Ex-post de la Iniciativa Comunitaria LEADER+ y de los Informes de Seguimiento del Programa PRODERCAL realizados entre los años 2003 y 2008, para la Dirección General de Infraestructuras y Diversificación Rural de la Junta de Castilla y León. Dichos Informes han sido dirigidos por el autor de este trabajo, con la colaboración de los siguientes miembros del Departamento de Economía Aplicada de la Universidad de Valladolid: Elena Fernández Alonso, Pablo de Frutos Madrazo, Inmaculada Mínguez Lara, Belén Miranda Escolar, Fernando Repiso Ortega, Ángel de los Ríos Rodicio, José Luis Rojo García, José Antonio Salvador Insúa e Isabel Vega Mocoroa.
} 
resultados que la aplicación de ambos Programas había tenido sobre las zonas rurales, analizando si se había conseguido cierto grado de revitalización de éstas, si se habían movilizado los recursos endógenos para generar riqueza y empleo, y si se percibía una mejora en la articulación social y económica sobre las comarcas destinatarias de las actuaciones. Asimismo, se trató de evaluar, también, la dinámica generada por la aplicación del "Método LEADER" que, básicamente, se refiere a la aplicación de un enfoque ascendente y participativo de la población en la estrategia de desarrollo, además de la coherencia, la transferibilidad y la innovación en su aplicación.

La existencia de 44 Grupos de Acción Local en el territorio regional, con una importante experiencia en acciones de desarrollo rural integradas que, en muchos casos, había ido desarrollándose a lo largo de más de doce años, tuvo importantes efectos positivos en la gestión de la Iniciativa LEADER+ y en el Programa PRODERCAL. La disposición de una estructura permanente de oficinas técnicas para el desarrollo rural, el conocimiento más cercano del territorio y de los agentes que en él operan, así como la experiencia en las relaciones con la Administración Regional constituyen un importante activo en esta región para el desarrollo de este tipo de programas.

En la ejecución de ambos Programas, tanto en los territorios LEADER+ como PRODERCAL, se ha podido observar una cierta mejora en las labores de difusión y dinamización realizadas por los $G A L$ en comparación con el período anterior. Sin embargo, se consideraba conveniente seguir impulsando este tipo de actividades durante los próximos años de manera que los equipos técnicos propiciaran la aparición de proyectos innovadores, de acuerdo con las características socioeconómicas del territorio y las posibles alternativas que pudieran presentarse para el mundo rural, ante los importantes cambios que, en aquel momento, la Comisión Europea tenía previsto introducir en la PAC y que iban a afectar seriamente a esta Comunidad Autónoma.

El conjunto de acciones desarrolladas a través de la Iniciativa LEADER+ y del Programa PRODERCAL, a partir del análisis de una muestra representativa de 624 proyectos que fueron evaluados directamente a través de las visitas realizadas a los beneficiarios, indicaban una clara correspondencia entre los proyectos financiados, el potencial endógeno de la zona y la estrategia establecida en los respectivos Planes de Desarrollo Local de cada Grupo. Asimismo, estos proyectos analizados, mostraron una elevada sostenibilidad económica aparente y un nivel medio en el grado de innovación y de capacidad demostrativa, siendo menor la diversificación económica que éstos habían introducido en los distintos territorios, especialmente en el caso de los proyectos acogidos al Programa PRODERCAL.

Las actividades que acapararon la mayor parte de las inversiones fueron: el apoyo a las PYMEs, el turismo rural, la valorización de los productos locales y 
los servicios a la población. Además, se constató que el grueso de las inversiones se concentraba en unos pocos lugares, dado que fueron los municipios que más gasto tenían asignado al principio del Programa, los que mostraron más capacidad de atracción de inversión y gasto al final. Por otro lado, cuando se consideraban las intervenciones en función del tamaño poblacional, se observaba que los municipios de mayor tamaño eran, también, los que acaparaban la mayor parte de las actuaciones y del gasto (Mapa 1). En consecuencia, la concentración detectada debe considerarse un resultado lógico, e incluso deseable, si con ello se logran consolidar algunos núcleos dinámicos capaces de proporcionar empleos y servicios en el entorno rural.

\section{Mapa 1}

Localización de las inversiones de los Programas $L E A D E R+$ y PRODERCAL en el territorio rural de Castilla y León (2003-2008)

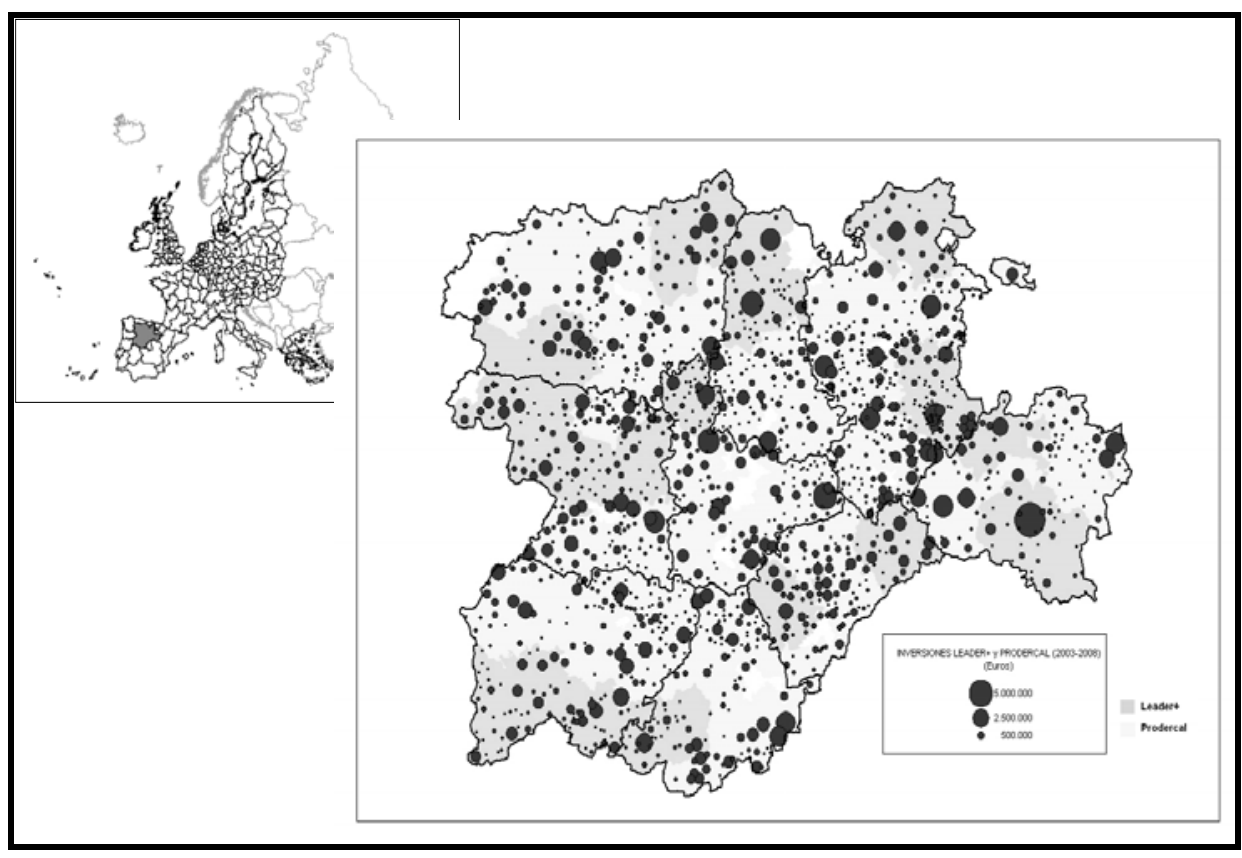

Fuente: Elaboración propia a partir de los datos procedentes del Informe de Evaluación Ex-post de la Iniciativa Comunitaria LEADER+ en Castilla y León y del Informe de Seguimiento del Programa PRODERCAL 2000-2006.

El empleo creció de manera sostenida a lo largo de la ejecución de estos dos Programas, alcanzando una cifra total de 3.457 nuevos puestos de trabajo, a los que habría que añadir otros 2.852 empleos mantenidos, contribuyendo de esta forma a lograr uno de sus objetivos más importantes, destacando especialmente el caso de las PYMEs, así como las actividades relacionadas con el turismo y 
con la transformación y comercialización de productos agroalimentarios. En todas ellas ha ido tomando cada vez mayor importancia la consolidación del empleo preexistente (Tablas 3 y 4 ).

Ahora bien, no todos los GAL mostraron la misma efectividad en el cumplimiento de este objetivo, situándose algunos de ellos muy por debajo de la media de cada uno de los Programas, si bien es cierto que la distinta caracterización socioeconómica de los espacios donde desarrollan su labor constituye un condicionante que no puede ser obviado; de hecho, los Grupos más exitosos están siempre situados en las comarcas rurales más dinámicas. En cualquier caso, es preciso tener presente que esta evolución tan positiva del empleo se vio influenciada favorablemente por el ciclo económico hasta la finalización de estos Programas, pero no cabe duda que, actualmente, las condiciones son menos favorables $\mathrm{y}$, tal como podremos apreciar a continuación, la situación es hoy mucho menos propicia para la creación de empleo en las zonas rurales.

Tabla 3

Creación y mantenimiento de empleo en Castilla y León por la aplicación de la Iniciativa Comunitaria LEADER+

\begin{tabular}{|c|c|c|c|c|}
\hline \multirow{12}{*}{$\begin{array}{l}\text { NÚMERO DE } \\
\text { EMPLEOS }\end{array}$} & \multirow{6}{*}{ CREADOS } & \multirow{3}{*}{ FIJOS } & TOTAL & 889 \\
\hline & & & MUJERES & 513 \\
\hline & & & $<40$ AÑOS & 556 \\
\hline & & \multirow{3}{*}{ EVENTUALES } & TOTAL & 473 \\
\hline & & & MUJERES & 264 \\
\hline & & & $<40$ AÑOS & 347 \\
\hline & \multirow{6}{*}{ MANTENIDOS } & \multirow{3}{*}{ FIJOS } & TOTAL & 1149 \\
\hline & & & MUJERES & 278 \\
\hline & & & $<40$ AÑOS & 547 \\
\hline & & \multirow{3}{*}{ EVENTUALES } & TOTAL & 257 \\
\hline & & & MUJERES & 78 \\
\hline & & & $<40$ AÑOS & 184 \\
\hline \multirow{2}{*}{\multicolumn{3}{|c|}{$\begin{array}{l}\text { EMPLEOS EN EMPRESAS DE COMERCIALIZACIÓN DE PRODUCTOS } \\
\text { AGRÍCOLAS DE CALIDAD }\end{array}$}} & CREADOS & 175 \\
\hline & & & MANTENIDOS & 226 \\
\hline \multirow{2}{*}{\multicolumn{3}{|c|}{ EMPLEOS DIRECTOS EN SECTOR TURÍSTICO }} & CREADOS & 309 \\
\hline & & & MANTENIDOS & 122 \\
\hline \multirow{2}{*}{\multicolumn{3}{|c|}{ EMPLEOS EN PYMES DE NUEVA CREACIÓN O AMPLIACIONES }} & CREADOS & 922 \\
\hline & & & MANTENIDOS & 1061 \\
\hline
\end{tabular}

Fuente: Datos procedentes del Informe de Evaluación Ex-post de la Iniciativa Comunitaria LEADER+ en Castilla y León, 2009. 
Tabla 4

Creación y mantenimiento de empleo en Castilla y León por la aplicación de Programa PRODERCAL

\begin{tabular}{|c|c|c|c|c|}
\hline \multirow{12}{*}{$\begin{array}{l}\text { NÚMERO DE } \\
\text { EMPLEOS }\end{array}$} & \multirow{6}{*}{ CREADOS } & \multirow{3}{*}{ FIJOS } & TOTAL & 1.429 \\
\hline & & & MUJERES & 775 \\
\hline & & & $<40$ AÑOS & 911 \\
\hline & & \multirow{3}{*}{ EVENTUALES } & TOTAL & 666 \\
\hline & & & MUJERES & 369 \\
\hline & & & $<40$ AÑOS & 426 \\
\hline & \multirow{6}{*}{ MANTENIDOS } & \multirow{3}{*}{ FIJOS } & TOTAL & 1.245 \\
\hline & & & MUJERES & 337 \\
\hline & & & $<40$ AÑOS & 662 \\
\hline & & \multirow{3}{*}{ EVENTUALES } & TOTAL & 201 \\
\hline & & & MUJERES & 82 \\
\hline & & & $<40$ AÑOS & 136 \\
\hline \multirow{2}{*}{\multicolumn{3}{|c|}{$\begin{array}{l}\text { EMPLEOS EN EMPRESAS DE COMERCIALIZACIÓN DE PRODUCTOS } \\
\text { AGRÍCOLAS DE CALIDAD }\end{array}$}} & CREADOS & 257 \\
\hline & & & MANTENIDOS & 285 \\
\hline \multirow{2}{*}{\multicolumn{3}{|c|}{ EMPLEOS DIRECTOS EN SECTOR TURÍSTICO }} & CREADOS & 635 \\
\hline & & & MANTENIDOS & 245 \\
\hline \multirow{2}{*}{\multicolumn{3}{|c|}{ EMPLEOS EN PYMES DE NUEVA CREACIÓN O AMPLIACIONES }} & CREADOS & 1.120 \\
\hline & & & MANTENIDOS & 1.155 \\
\hline
\end{tabular}

Fuente: Datos procedentes del Informe de Seguimiento del Programa PRODERCAL en Castilla y León, 2009.

En la Iniciativa LEADER+ y el Programa PRODERCAL de la región ha sido siempre muy relevante la presencia de operadores públicos, principalmente ayuntamientos, en los órganos de representación y decisión de los Grupos de Acción Local, en relación con la participación de personas físicas y colectivos empresariales. En este sentido, se considera que esta circunstancia ha incidido claramente en el mayor peso que en estos Programas representan las acciones cuyos beneficiarios son las entidades locales, dadas las importantes necesidades de financiación que éstas tienen para dotar de servicios básicos a la población. Esta orientación de los recursos ha significado una menor incidencia de la estrategia propuesta por los GAL dirigida principalmente a la reconversión productiva del medio rural.

Para el conjunto de los Grupos, una de las asignaturas pendientes ha sido la cooperación territorial, más allá de Castilla y León, así como la integración en red con otros socios. Así, se considera que, dada la experiencia acumulada en el tiempo por la aplicación del "Método LEADER", los resultados obtenidos deberían haber sido más satisfactorios, a pesar de que la mayoría de los $G A L$ ha logrado aumentar el número de proyectos y actividades de cooperación. En este sentido, dado el interés y la innovación que pueden suponer este tipo de acciones para el desarrollo futuro del territorio rural y la experiencia adquirida en los últimos años, los Grupos debieran dedicar más atención y continuidad a 
promocionar estas actividades, solicitando de las distintas administraciones, si fuera preciso, una mayor colaboración para solucionar los problemas que puedan derivarse de la elaboración y tramitación de los expedientes.

\subsection{Primeros resultados del Eje 4: LEADER del Programa de Desarrollo Rural de Castilla y León. Período 2007-2013}

Durante el período de programación actual, según queda reflejado en las "Directrices Estratégicas de Desarrollo Rural 2007-2013", , cada Estado Miembro presentó su plan nacional de estrategias de desarrollo rural, que constituye el marco de referencia para la preparación de los Programas de Desarrollo Rural $^{9}$, estando los recursos asignados a las prioridades comunitarias en esta materia en función de la evaluación específica de los puntos fuertes y débiles de cada zona. Estas Directrices, tal como se establece en el citado Documento, son las siguientes:

- Mejora de la competitividad en los sectores agrario y silvícola (Eje 1). Los sectores agrario, silvícola y de transformación alimentaria disponen de un gran potencial para ampliar la gama de los productos de gran calidad y con un gran valor añadido, tal como se viene demandando con mayor intensidad en los mercados europeos y mundiales. Los recursos destinados a este Eje 1 deben contribuir a consolidar un sector agroalimentario europeo fuerte y dinámico.

- Mejora del medio ambiente y del entorno natural (Eje 2). Al objeto de proteger y mejorar los recursos naturales y los paisajes de las zonas rurales de la Unión Europea, los fondos que se asignen a este Eje 2 deben utilizarse en los ámbitos de: la biodiversidad; la preservación y el desarrollo de los sistemas agrarios $y$ forestales de gran valor medioambiental y de los paisajes agrarios tradicionales; el agua; $\mathrm{y}$, el cambio climático.

- Mejora de la calidad de vida en las zonas rurales y fomento de la diversificación de la economía rural (Eje 3). Los recursos que se asignen a este Eje 3, deben contribuir a la creación de oportunidades de empleo y condiciones para el crecimiento. Para ello se debe fomentar la capacitación, la adquisición de cualificaciones y una organización

\footnotetext{
${ }^{8}$ Decisión del Consejo de 20 de febrero de 2006 sobre las directrices estratégicas comunitarias de desarrollo rural (período de programación 2007-2013) (2006/144/CE). Diario Oficial de la Unión Europea 25.2.2006.

${ }^{9}$ El Estado Miembro puede presentar un programa único para todo su territorio o un conjunto de programas regionales. España presentó para su aprobación 17 Programas de Desarrollo Rural, uno por cada Comunidad Autónoma, y un Programa Nacional, el Programa de la Red Rural Nacional. Todos ellos fueron aprobados por la Comisión Europea y actualmente se encuentran en fase de ejecución.
} 
adecuada que permita aplicar estrategias locales en las correspondientes zonas rurales ${ }^{10}$.

- Desarrollo de la capacidad local de creación de empleo y diversificación (Eje 4.LEADER). Los fondos que se destinen a este Eje deberán contribuir a la consecución de los objetivos prioritarios contemplados en el resto de los Ejes, además de facilitar la forma de organizarse y movilizar el potencial de desarrollo endógeno de las zonas rurales.

Estas Directrices se han plasmado, en esta Comunidad Autónoma, en el Programa de Desarrollo Rural 2007-2013, ejecutándose actualmente a través de 21 medidas distribuidas entre esos cuatro Ejes.

Corresponde analizar en este punto los primeros resultados del Eje 4.LEADER, al que se ha asignado, inicialmente, el 10\% de los recursos públicos del conjunto del Programa de Desarrollo Rural, y que en Castilla y León se está aplicando a través del Programa LEADERCAL (Mapa 2). Con él se pretende mantener una decidida continuidad con las experiencias de este tipo, iniciadas y desarrolladas con relativo éxito en Castilla y León desde hace dos décadas, a través de las Iniciativas Comunitarias LEADER I, LEADER II y $L E A D E R+$.

Del total de inversiones previstas en el Programa LEADERCAL (Tabla 5), las referentes a las estrategias de desarrollo local (medida 413) suponen el $82 \%$ de los recursos públicos previstos para la totalidad del Eje 4.LEADER. Hasta el momento, el proceso de evaluación continua del Programa de Desarrollo Rural de Castilla y León 2007-2013, ha puesto de manifiesto que el conjunto de acciones contempladas en esta medida está logrando movilizar el potencial endógeno de las zonas rurales de la región, diversificando su economía y contribuyendo positivamente a una mejora de la calidad de vida y de su entorno natural.

${ }^{10}$ Asimismo se indica que cuando se promueva la formación, información y espíritu empresarial se deberá prestar especial atención a las necesidades específicas de mujeres, jóvenes y trabajadores de edad avanzada. 


\section{Mapa 2}

Territorio de los 44 Grupos de Acción Local que participan en el Programa LEADERCAL en Castilla y León (2007-2013)

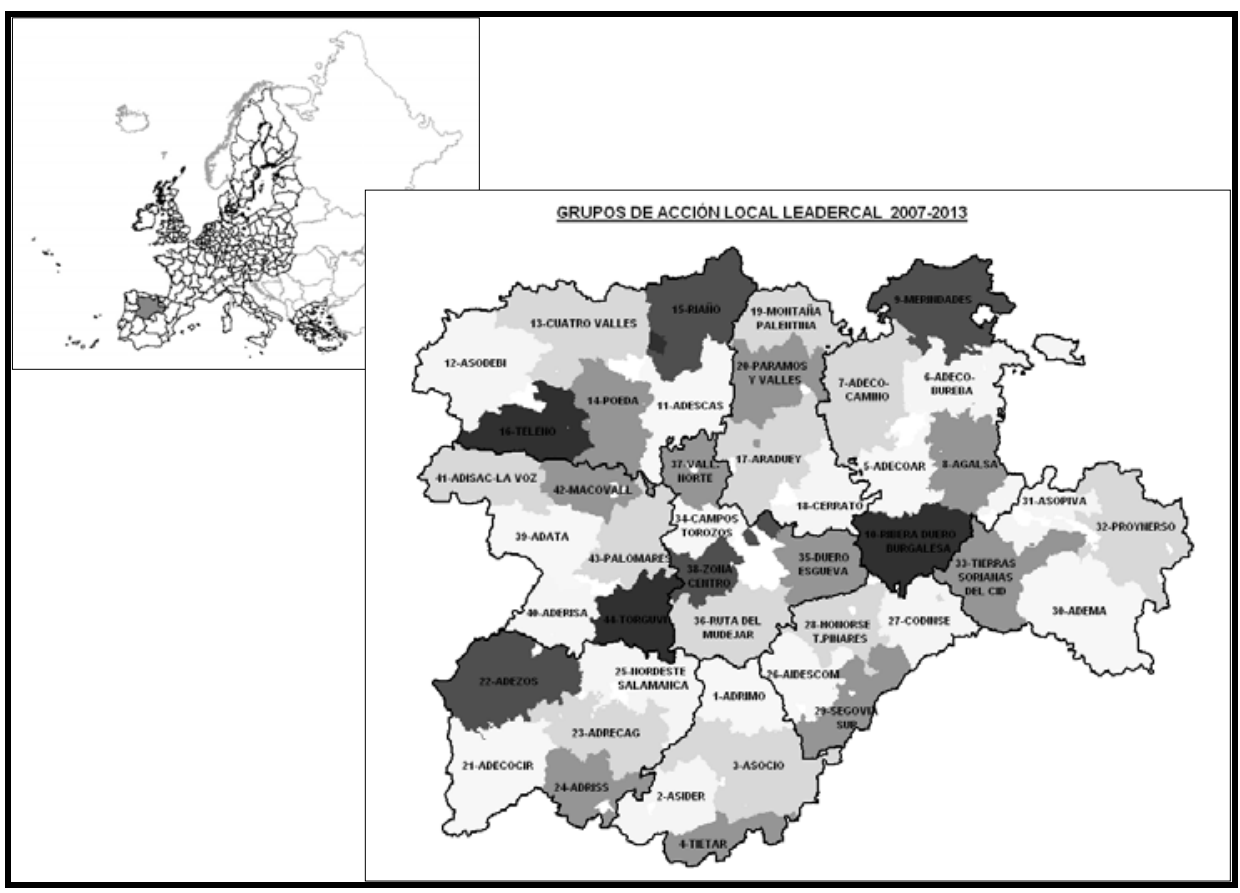

Fuente: Elaboración propia a partir de los datos procedentes de la Dirección General de Infraestructuras y Diversificación Rural. Consejería de Agricultura y Ganadería. Junta de Castilla y León.

Tabla 5

Inversión y gasto público previsto en el Programa LEADERCAL por medidas para el conjunto del período 2007-2013

\begin{tabular}{|c|c|c|c|c|c|c|c|c|}
\hline \multirow[b]{2}{*}{ MEDIDAS } & \multirow{2}{*}{$\begin{array}{c}\text { TOTAL } \\
\text { PUBLICO } \\
(1)=(2)+(4)\end{array}$} & \multicolumn{2}{|c|}{ FONDOS EUROPEOS } & \multicolumn{4}{|c|}{ FONDOS NACIONALES PROGRAMA } & \multirow{2}{*}{$\begin{array}{l}\text { APORTACIÓN } \\
\text { PRIVADA } \\
\text { (8) }\end{array}$} \\
\hline & & $\begin{array}{l}\text { FEADER } \\
\text { (2) }\end{array}$ & $\begin{array}{c}\% \\
(3)=(2) /(1)\end{array}$ & $\begin{array}{c}\text { TOTAL } \\
(4)=(6)+(7)\end{array}$ & $\begin{array}{c}\% \\
(5)=(4) /(1)\end{array}$ & $\begin{array}{l}\text { CENTRAL } \\
\text { (6) }\end{array}$ & $\begin{array}{l}\text { AUTONÓMICA } \\
\text { (7) }\end{array}$ & \\
\hline $\begin{array}{l}\text { 413: ESTRATÉGIAS DE } \\
\text { DESARROLLO LOCAL }\end{array}$ & 175.600 .000 & 61.460 .000 & 35,00 & 114.140 .000 & 65,00 & 57.070 .000 & 57.070 .000 & 175.600 .000 \\
\hline $\begin{array}{l}\text { 421: COOPERACIÓN } \\
\text { TERRITORIAL }\end{array}$ & 7.000 .000 & 2.450 .000 & 35,00 & 4.550 .000 & 65,00 & 2.275 .000 & 2.275 .000 & 0 \\
\hline $\begin{array}{l}\text { 431: } \\
\text { FUNCIONAMIENTO Y } \\
\text { ADQ. CAPACIDADES }\end{array}$ & 32.228 .571 & 11.280 .000 & 35,00 & 20.948 .571 & 65,00 & 10.474 .286 & 10.474 .285 & 0 \\
\hline TOTAL & 214.828 .571 & 75.190 .000 & 35,00 & 139.638 .571 & 65,00 & 69.819 .286 & 69.819 .285 & 175.600 .000 \\
\hline
\end{tabular}

Fuente: Dirección General de Infraestructuras y Diversificación Rural. Consejería de Agricultura y Ganadería. Junta de Castilla y León. 
Asimismo, ha podido apreciarse un cierto retraso en la ejecución financiera debido, en gran parte, a la repercusión que la crisis económica está teniendo también sobre la actividad productiva de las áreas rurales. Al analizar el volumen de recursos ejecutados en esta medida 413, hasta la primera mitad del año 2010, éstos apenas alcanzaban los 90 millones de euros, una cantidad inferior a la prevista en el Programa para los tres primeros años de ejecución (2007, 2008 y 2009). Cabe resaltar, además, que una gran parte de las inversiones están siendo destinadas a las acciones 312, 313 y 321 (Tabla 6) y que el número de empleos que figuraban en los expedientes aceptados, hasta el momento de cierre de la evaluación (junio de 2010), están todavía en pleno proceso de ejecución y consolidación. De este modo, la posible creación de 585 empleos (5,6 puestos de trabajo por cada 10.000 habitantes) supondría una inversión media global próxima a los 170.000 euros por cada nuevo puesto de trabajo creado.

Tabla 6

Efectos de la aplicación de las estrategias de desarrollo local a través del Enfoque LEADER. Medida 413 del Programa de Desarrollo Rural de Castilla y León 2007-2013 (*)

\begin{tabular}{|l|c|c|c|c|}
\hline \multicolumn{1}{|c|}{ Acción } & Acciones & Inversión & $\begin{array}{c}\text { Empleo } \\
\text { creado }\end{array}$ & $\begin{array}{c}\text { Empleo } \\
\text { mantenido }\end{array}$ \\
\hline $\begin{array}{l}\text { 413 - Estrategias de desarrollo local a través } \\
\text { del enfoque LEADER }\end{array}$ & $\mathbf{8 1 8}$ & $\mathbf{8 9 . 3 3 1 . 5 6 0}$ & $\mathbf{5 8 5}$ & $\mathbf{4 2 9}$ \\
\hline $\begin{array}{l}\text { 311. Diversificación hacia actividades no } \\
\text { agrícolas. }\end{array}$ & 6 & 1.089 .892 & 5 & 4 \\
\hline $\begin{array}{l}\text { 312. Ayuda a la creación y el desarrollo de } \\
\text { microempresas. }\end{array}$ & 225 & 29.045 .017 & 303 & 224 \\
\hline 313. Fomento de actividades turísticas & 193 & 31.162 .555 & 186 & 120 \\
\hline $\begin{array}{l}\text { 321. Prestación de servicios básicos para la } \\
\text { economía y la población rural }\end{array}$ & 144 & 14.074 .291 & 71 & 69 \\
\hline $\begin{array}{l}\text { 322. Renovación y desarrollo de poblaciones } \\
\text { rurales }\end{array}$ & 51 & 4.585 .100 & 17 & 0 \\
\hline 323. Conservación y mejora del patrimonio rural & 181 & 7.340 .412 & 3 & 12 \\
\hline $\begin{array}{l}\text { 333. Formación e información de los agentes } \\
\text { económicos }\end{array}$ & 18 & 300.494 & 0 & 0 \\
\hline
\end{tabular}

(*) Número de acciones e inversiones que figuran en los Registros de Expedientes de los Grupos de Acción Local y estimación de los puestos de trabajo creados y mantenidos. Datos registrados hasta junio de 2010.

Fuente: Datos procedentes del Informe de Evaluación a Medio Plazo del Programa de Desarrollo Rural de Castilla y León, 2007-2013 realizado en 2010.

La distribución de las inversiones realizadas muestra que al menos 6 GAL apenas habían iniciado los proyectos que configuran la estrategia de desarrollo local en sus respectivos territorios; un número similar de Grupos ha concentrado sus escasas inversiones en un número muy reducido de municipios. 
En el resto de las comarcas donde la cuantía de las inversiones es algo más elevada, en relación con las anteriores, puede comprobarse también la fuerte concentración de éstas en unos pocos municipios. Se trata de una circunstancia que sigue constituyendo una de las características esenciales en la aplicación de los anteriores Programas LEADER puestos en marcha de forma generalizada en la región desde los años 90; lo cual parece que viene a confirmar nuevamente la existencia de grandes espacios rurales en Castilla y León que, por su estructura socioeconómica, no están siendo capaces de presentar condiciones favorables para desarrollar con éxito estrategias de desarrollo y diversificación de la economía rural.

En el Programa LEADERCAL, el conjunto de actividades desarrolladas por los responsables del funcionamiento de los $G A L$, relacionadas con la promoción del enfoque multisectorial y la cooperación entre los territorios, no puede considerarse hasta ahora relevante, a juzgar por el número y cuantía asignada a este tipo de acciones, tanto en términos absolutos como en relación a la población rural. No obstante, debe tenerse en cuenta que la promoción en las zonas rurales de Castilla y León es una labor que ha venido desarrollándose con cierta intensidad a lo largo de los anteriores Programas puestos en marcha en la región, bajo el enfoque $L E A D E R$, a partir de 1995; esta circunstancia parece haber influido decisivamente en el hecho de que la práctica totalidad de los Grupos, durante el actual período de programación, no hayan considerado prioritaria su difusión entre los agentes sociales del territorio.

El Eje 4.LEADER está teniendo hasta ahora una escasa incidencia en el sector agrario regional. Esta circunstancia se debe principalmente a la escasa (nula) presencia de cursos de formación que hayan podido incidir en la modernización del sector y también a la menor presencia de proyectos que hayan podido repercutir en una mejora en la calidad de los productos agrarios. Asimismo, otro de los factores responsables de este resultado es la ausencia de financiación en este Eje 4, hasta ahora, para las industrias alimentarias, que son una de las claves para propiciar la transformación y modernización del sector agrario. No obstante, recientemente han sido admitidas a la financiación en este Eje aquellas actividades de transformación alimentaria cuyo volumen de inversión no supere los 25.000 euros.

Los proyectos de cooperación se están orientando principalmente al sector turístico, y la práctica totalidad de los proyectos de transferencia de "buenas prácticas" están dirigidos, en su conjunto, a la mejora de la calidad de vida en las zonas rurales y a la diversificación de la economía rural, en especial aquellas acciones referidas a la creación de microempresas, fomento de actividades 
turísticas, prestación de servicios a la población y conservación del patrimonio rural $^{11}$.

Finalmente, cabe resaltar, como sucedía en los períodos analizados anteriormente, el elevado protagonismo de las corporaciones locales en el funcionamiento de los Grupos de Acción Local, así como en la aplicación de su estrategia de desarrollo, lo cual hace posible la puesta en marcha de diversas actividades no productivas, financiadas a través del Eje 4.LEADER, con la finalidad de captar recursos públicos para cubrir proyectos sociales que no pueden atender con sus presupuestos ordinarios, especialmente en unos años en los que se tienen previstos importantes recortes financieros en sus cuentas públicas.

\section{CONSIDERACIONES FINALES}

No cabe duda que la experiencia en Castilla y León sobre la aplicación de este tipo de políticas que acabamos de analizar está resultando bastante positiva, en lo que se refiere a su contribución a la transformación de la actividad socioeconómica de su espacio rural, tal como viene exigiendo la evolución del sector agrario ante los procesos de integración europea y globalización del comercio mundial. En los trabajos de evaluación y seguimiento de estos Programas, realizados por nuestro equipo para el gobierno regional desde 1997, hemos podido apreciar que, efectivamente, se ha iniciado un proceso interesante de reconversión tan necesario para el mundo agrario tradicional que, aunque tarde en su aplicación, debería continuar con mucha mayor intensidad en el futuro. Sin embargo, una de las claves que, a nuestro juicio, están retardando este urgente proceso del mundo rural, orientado a la utilización del suelo para productos y servicios distintos a los tradicionales, es la escasez de recursos destinados a este tipo de actuaciones, si tenemos en cuenta la cuantía de estas ayudas en comparación con el conjunto de subvenciones directas destinadas al sector agrario a través de la PAC (Primer pilar), puesto que los recursos dedicados al desarrollo rural en sus diversas vertientes (no sólo considerando la política territorial aquí analizada, sino aquellas otras actuaciones orientadas a conseguir reformas estructurales en el sector, como la modernización de explotaciones y una mayor cualificación del capital humano) han supuesto, aproximadamente, tan solo el $10 \%$ de los fondos destinados a la PAC en los diferentes períodos analizados.

11 La valoración que los técnicos de la administración regional han efectuado sobre la contribución del Eje 4.LEADER a la transferencia de "buenas prácticas" ha sido muy positiva, puesto que ha alcanzado una calificación global de 4 puntos sobre una escala de baremación de 0 a 5. 
Las políticas de desarrollo rural, orientadas especialmente a promover en el espacio agrario actividades económicas distintas a las tradicionales, seguirán constituyendo uno de los aspectos clave de la futura PAC y pueden apoyar la necesaria reconversión del sector agrario en el horizonte 2020. Sin embargo, tal como se ha indicado, el amplio territorio rural de Castilla y León presenta unas características socioeconómicas bastante diferenciadas, de forma que más de las tres cuartas partes de los núcleos de población muestran, en principio, condiciones muy poco favorables para el mantenimiento de la población y el impulso del desarrollo endógeno, a través de la diversificación $\mathrm{y}$ reestructuración de su sector agrario.

Resulta difícil llegar a establecer con precisión valoraciones cuantitativas sobre la repercusión que están teniendo las políticas territoriales de desarrollo rural puestas en marcha en esta Comunidad Autónoma a partir de 1991 con las diversas etapas llevadas a cabo en las Iniciativas LEADER y los Programas $P R O D E R$, sobre todo por el escaso volumen de recursos financieros empleados, con relación al resto de las políticas aplicadas en las zonas rurales. Sin embargo, no cabe duda que en los citados programas se ha podido observar, en general, un cierto grado de coherencia en las estrategias de desarrollo planteadas, con relación a las potencialidades de los distintos territorios; debe resaltarse además el hecho de que las acciones realizadas no resultaron todo lo innovadoras que se pretendía, si bien han contribuido claramente a iniciar como ejemplos interesantes a seguir, una serie de cambios de orientación productiva en el mundo rural que pueden ser el comienzo de una necesaria reconversión en todo el territorio.

Los proyectos financiados a través de estos Programas, hasta la actualidad, han presentado un cierto grado de sostenibilidad económica y han propiciado la creación y el mantenimiento en el medio rural de más de 7.500 puestos de trabajo, una gran parte de ellos fijos, que incluyen una elevada proporción de mujeres y jóvenes. Los resultados han tenido especial repercusión en el aprovechamiento de las potencialidades turísticas y en el incremento de competitividad de numerosas empresas que han ampliado su actividad en el medio rural, especialmente la relacionada con la transformación $\mathrm{y}$ comercialización de productos agrarios.

Debe considerarse, además, bastante positiva la acción de los Grupos en el territorio, ya que, en principio, supone la presencia en el medio rural de oficinas técnicas de apoyo al desarrollo y grupos de dinamización que contribuyen a mejorar la capacidad organizativa. No obstante, la influencia de las administraciones públicas en estos Grupos, aunque limitada por ley, puede considerarse aún elevada para en un tipo de proyectos económicos cuya base es la iniciativa privada. Consecuentemente, el enfoque LEADER es un importante activo generado en las áreas rurales de la región desde hace más de dos décadas 
que ha contribuido positivamente a su gobernanza, además de establecer una serie de vínculos estables entre la administración y los ciudadanos para canalizar propuestas específicas de desarrollo en su territorio.

Por otro lado, no conviene olvidar el hecho de que las inversiones más significativas propiciadas por estos Programas se estén localizando en un reducido número de municipios, caracterizados por su gran tamaño y por una dinámica económica propia bastante positiva, frente a la dispersión y menor cuantía de las acciones financiadas en los núcleos rurales menos desarrollados de la región. Este hecho nos lleva a plantearnos la capacidad de este tipo de políticas territoriales para reducir los desequilibrios del espacio rural de Castilla y León, dadas las grandes diferencias actualmente existentes en las distintas zonas.

Finalmente, ante los escasos recursos previstos para reforzar este tipo de iniciativas en el mundo rural, ya sea por el lento proceso de modulación en la PAC, o porque Castilla y León ya no goza de las ventajas de ser considerada prioritaria a los efectos de la política de cohesión, no parece que este interesante planteamiento para la transformación rural vaya a recibir un impulso decisivo en el futuro. De hecho, la proporción de recursos destinados actualmente a las políticas territoriales de desarrollo rural en la región solamente alcanza el 10\% de los recursos públicos previstos en el Programa de Desarrollo Rural de Castilla y León 2007-2013, en el cual se contemplan también importantes ayudas orientadas a la modernización de las explotaciones y a la mejora de regadíos, entre otras. Se echa en falta, por tanto, una mayor dotación de recursos que faciliten una auténtica reconversión del sector hacia otras actividades distintas a las tradicionales, en función de la creciente demanda que tienen por parte de la sociedad, tanto en términos medioambientales y transformaciones alimentarias de calidad, como de servicios de ocio y esparcimiento.

\section{REFERENCIAS BIBLIOGRÁFICAS}

CONSEJO EUROPEO (2006): Decisión de 20 de febrero sobre las Directrices Estratégicas Comunitarias de Desarrollo Rural (período de programación 20072013). Diario Oficial de la Unión Europea, 25 de febrero de 2006.

FARIÑAS GÓMEZ, B.; GORDO GÓMEZ, P.; RÍOS RODICIO, A. y RODRIGUEZ PRADO, B. (2004): Identificación y tipificación de los espacios rurales en la Comunidad Autónoma de Castilla y León. Una aproximación para el análisis de las dinámicas espaciales. Valladolid. Junta de Castilla y León.

GARCIA VILLAREJO, A.; NOGALES GARCÍA, J. y RIOS RODICIO, A. (2005): "Incidencia de las ayudas europeas en Castilla y León y retos que presenta el nuevo escenario de las perspectivas financieras para el período 2007-2013". En Vega 
Mocoroa, I. (Coord.): Logros, iniciativas y retos institucionales y económicos: La Unión Europea del siglo XXI. Valladolid. Lex Nova.

GORDO GÓMEZ, P. (Dir.) y OTROS (2001): Evaluación "Ex-post" de la ejecución del Programa Regional de Castilla y León en la Iniciativa Comunitaria LEADER II (19951999). Departamento de Economía Aplicada. Universidad de Valladolid. Mimeo.

GORDO GÓMEZ, P. (2005): "Repercusión de las políticas territoriales de desarrollo rural en Castilla y León: Iniciativa Comunitaria LEADER+ y Programa PRODERCAL (2000-2006)". En Vega Mocoroa, I. (Coord.): Logros, iniciativas y retos institucionales y económicos: La Unión Europea del siglo XXI. Valladolid. Lex Nova.

GORDO GÓMEZ, P. (2007): "Perspectivas económicas de los espacios rurales de Castilla y León: Experiencia de las políticas territoriales de desarrollo rural aplicadas y propuestas para el período 2007-2013". En: Instituto de Estudios Europeos (Ed.): Tratado de Roma en su 50 aniversario (1957-2007). Balance socioeconómico de la Unión Europea (pp.687-708). Valladolid. Universidad de Valladolid.

GORDO GÓMEZ, P. (Dir.) y OTROS (2008): Evaluación Ex-post de la Iniciativa Comunitaria LEADER+ en Castilla y León e Informe de Seguimiento del Programa PRODERCAL 2000-2006. Departamento de Economía Aplicada. Universidad de Valladolid. Mimeo.

MIRANDA ESCOLAR, B. (Dir.) y OTROS (2010): Informe de Evaluación a Medio Plazo del Programa de Desarrollo Rural de Castilla y León 2007-2013. Departamento de Economía Aplicada. Universidad de Valladolid. Mimeo. 